Japan. J. Genetics Vol. 51, No. 5: 305-314 (1976)

\title{
EFFECTS OF POPULATION DENSITY AND TEMPERATURE CONDITION ON FITNESS IN DROSOPHILA MELANOGASTER \\ II. FECUNDITY AND MORTALITY
}

\author{
SEIDO OHNISNI
}

National Institute of Genetics, Mishima 411

Received March 19, 1976

It is well known that one component of fitness in Drosophila, pre-adult viability, varies with larval density (Lewontin 1955; Lewontin and Matsuo 1963), nutritional condition (Ohba 1961; Palabost 1972) and temperature (Parsons 1959; Beardmore and Levine 1963; Dawood and Strickberger 1964; Young 1970). In addition, another pre-adult fitness component, depelopmental time, is influenced by larval density and temperature (Ohnishi 1976).

Though the effects of various environments on pre-adult fitness components have been extensively studied, their effects on fecundity (egg production) have been largely neglected, in spite of the important contribution of fecundity to total fitness. Furuya and Mori (1961) reported thet the number of eggs laid by adult flies was affected by adult density and by nutritional conditions. Also, Oshima and Kawanishi (1969) showed that mean number of eggs was smaller under a fluctuating temperature than under a constant one. In this vein, Oshima et al. (1972), Oshima and Choo (1971) and Choo and Oshima (1974) reported that fecundity or oviposition pattern was strongly affected by light conditions. Moreover, longevity, which is closely related to fecundity, was greater under fluctuating light conditions than under constant ones (Erk and Samis 1970).

The present study was mainly designed to investigate how fecundity (mean number of eggs per female per day for 20 days) is influenced by adult density and fluctuating temperature. In addition, the relationship of fecundity to mortality was studied under various environmental conditions by correlation analysis.

\section{MATERIALS AND METHODS}

Many males of Drosophila melanogaster were sampled from a natural population of Kofu, Yamanashi-ken, Japan, in the fall of 1972 and a second chromosome was extracted from each fly by the $C y$-method. From the chromosomes in the sample which were free of viability-reducing genes, two were randomly chosen; these were designated as strains $10 \mathrm{~A}$ and $22 \mathrm{~B}$. In this experiment, the fecundity of homozygous $(+/+)$ and heterozygous $(C y /+)$ flies obtained from the offspring of each of these strains was estimated under various conditions.

Adult pairs (one day old) were put into a small vial (about $9 \mathrm{~cm}$ in length, having 
diameter $2 \mathrm{~cm}$ ) filled with about $5 \mathrm{ml}$ of medium. The number of adult pairs introduced into the vial was one, two or eight, correspond to three levels of adult density. The crosses (five replications of each density) were carried out under both constant $\left(25^{\circ} \mathrm{C}\right)$ and fluctuating (a $20^{\circ} \mathrm{C} \sim 30^{\circ} \mathrm{C}$ cycle per day) temperatures. A total of 120 crosses were performed simultaneously. For 20 days, the adult pairs were transferred into new vials every day and the eggs which were laid on the medium were counted every day. During the experiment flies which died were not replaced with fresh ones; since 20-day mortality was low, it was neglected in calculating the mean number of eggs per female. In order to examine mortality, after the 20 day egg counting period, the adult pairs were successively transferred into new vials every two or three days; the number of living fles was recorded periodically. The experiment was performed under constant illumination (about 700 lux).

\section{RESULTS AND ANALYSES}

Oviposition pattern: Eggs laid on the culture medium of each vial were counted every day for 20 days. The mean number of eggs laid by a homozygous or heterozygous female of each strain was calculated for each day; the relevant time plots (oviposition curves) are presented in Figs. 1 and 2.

\section{A-Strain}

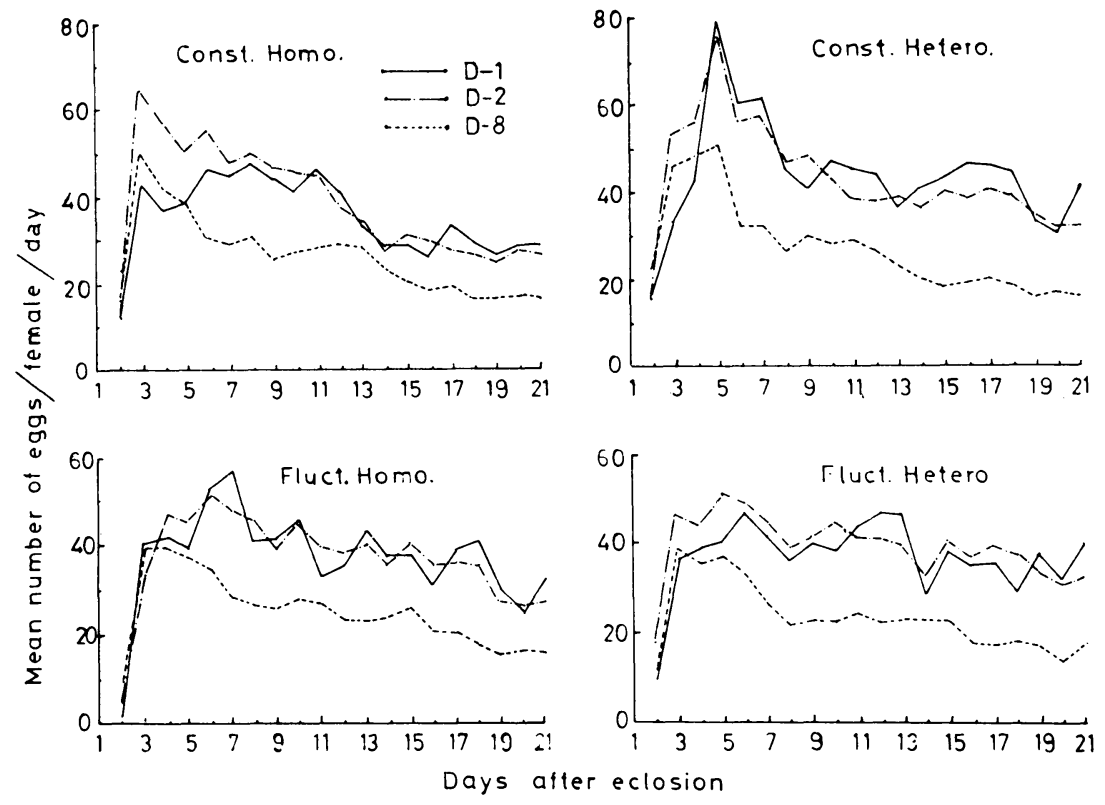

Fig. 1. Oviposition pattern of homozygous and heterozygous flies of strain $10 \mathrm{~A}$ in three levels of density under constant $\left(25^{\circ} \mathrm{C}\right.$ ) and fluctuating (a $20^{\circ} \mathrm{C} \sim 30^{\circ} \mathrm{C}$ cycle per day) temperatures. Solid, dot-dashed and broken lines show mean number of eggs laid per female per day in one, two and eight female density levels, respectively. 


\section{B-Strain}

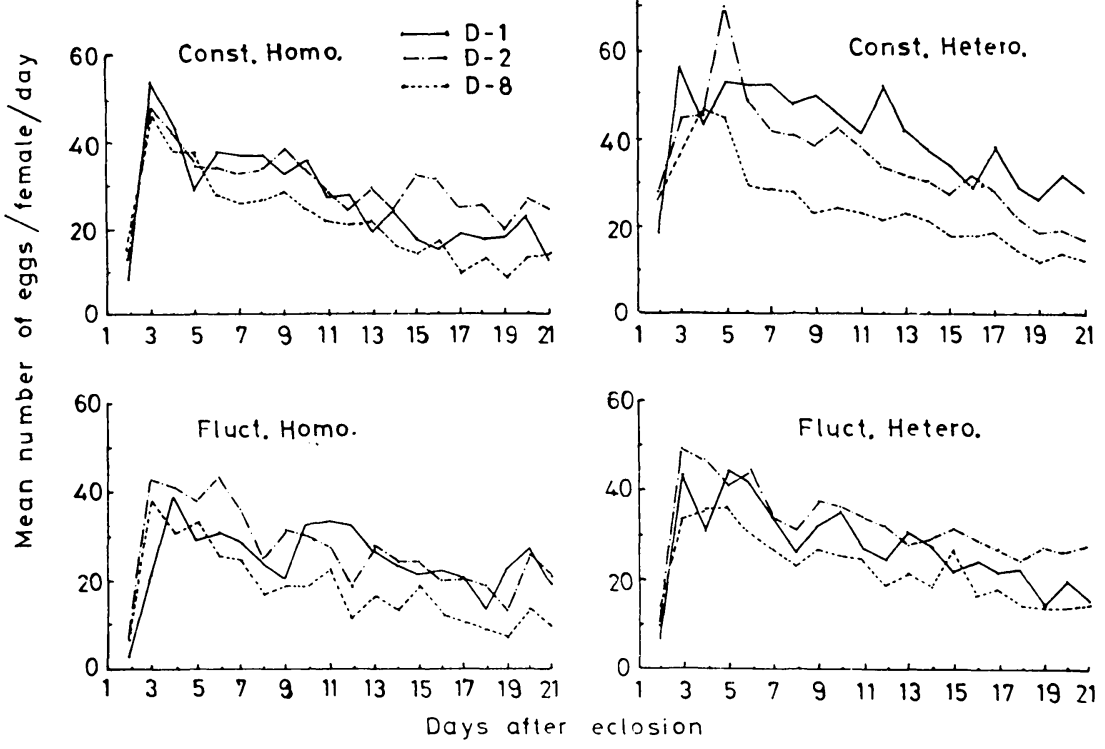

Fig. 2. Oviposition pattern of homozygous and heterozygous flies of strain 22B. Other comments as for Fig. 1.

These oviposition curves show an adult density effect for fecundity for both chromosome types (homozygotes and heterozygotes) of the two strains; namely the mean number of eggs laid per female in crowded conditions decreased markedly as compared with that in uncrowded conditions with either chromosome type of either strain. The trend was observed under both temperature conditions. The oviposition patterns under the fluctuating temperature were found to be flatter than those under the constant one.

Mean number of eggs per female per day: The mean number of eggs laid per female per day for 20 days by a homozygous or heterozygous fly of strains $10 \mathrm{~A}$ and $22 \mathrm{~B}$ was calculated for three levels of adult density under the constant and fluctuating temperatures. The results are given in Table 1 .

In all cases, the mean number of eggs laid per female per day in the eight female density class was lower than that in the one or two female density class (Table 1, Figs. 1 and 2). The mean number of eggs under the fluctuating temperature also decreased as compared with that under the constant one. Moreover, the mean number of eggs laid by heterozygous females was larger than that laid by homozygous ones under almost all conditions. The mean number of eggs laid by heterozygous females of strain $22 \mathrm{~B}$ was exceptionally large in the one pair density experiment with constant temperature (40.23). The reason for this is unknown.

Analysis of variance for fecundity was carried out, using the mean number of eggs laid for 20 days as data. Because the variances were significantly different among adult densities, analyses were carried out separately at each density level. The results are given in Table 2. 
Table 1. Mean number of eggs laid per female per day for 20 days by homozygous or heterozygous females of strains $10 \mathrm{~A}$ and $22 \mathrm{~B}$ in three density levels under constant and fluctuating temperatures

\begin{tabular}{|c|c|c|c|c|c|}
\hline \multirow{2}{*}{ Strain } & \multirow{2}{*}{ Density } & \multicolumn{2}{|c|}{ Constant } & \multicolumn{2}{|c|}{ Fluctuating } \\
\hline & & Homo & Hetero & Homo & Hetero \\
\hline $10 \mathrm{~A}$ & 우 $1 \times \hat{o} 1$ & $32.25 \pm 2.51 *$ & $43.76 \pm 2.43$ & $36.90 \pm 1.88$ & $37.33 \pm 2.42$ \\
\hline " & $2 \times \quad 2$ & $38.21 \pm 3.16$ & $43.00 \pm 1.65$ & $36.90 \pm 4.08$ & $38.81 \pm 1.37$ \\
\hline " & $8 \times 8$ & $25.98 \pm 1.38$ & $26.94 \pm 0.80$ & $24.74 \pm 1.05$ & $22.95 \pm 0.92$ \\
\hline $22 \mathrm{~B}$ & 우 $1 \times \hat{\delta} 1$ & $26.72 \pm 6.62$ & $40.23 \pm 2.74$ & $24.41 \pm 3.49$ & $27.09 \pm 2.93$ \\
\hline$" \prime$ & $2 \times \quad 2$ & $29.59 \pm 4.04$ & $34.54 \pm 1.23$ & $25.87 \pm 2.79$ & $32.06 \pm 2.27$ \\
\hline " & $8 \times 8$ & $21.92 \pm 1.38$ & $23.72 \pm 0.51$ & $18.15 \pm 1.25$ & $22.24 \pm 0.80$ \\
\hline
\end{tabular}

* Standard error.

Table 2. Analysis of variance for fecundity (mean number of eggs per female per day for 20 days) at each density level

\begin{tabular}{|c|c|c|c|c|c|c|c|}
\hline \multirow{2}{*}{$\begin{array}{l}\text { Source of } \\
\text { variation }\end{array}$} & \multirow{2}{*}{$\begin{array}{l}\text { Degree of } \\
\text { freedom }\end{array}$} & \multicolumn{2}{|c|}{ Density-1a } & \multicolumn{2}{|c|}{ Density-2a } & \multicolumn{2}{|c|}{ Density- $8^{a}$} \\
\hline & & M.S. & $F$-value & M.S. & $F$-value & M.S. & $F$-value \\
\hline Strain $(\mathrm{S})$ & 1 & 756.47 & $12.91^{* *}$ & 767.38 & $18.79 * *$ & 132.78 & $25.15^{* *}$ \\
\hline Chromosome type & (C) & 394.70 & $6.74^{*}$ & 194.04 & $4.75^{*}$ & 16.00 & 3.03 \\
\hline Temperature $(\mathrm{T})$ & 1 & 255.78 & $4.37 *$ & 82.60 & 2.02 & 68.59 & $12.99 * *$ \\
\hline $\mathrm{S} \times \mathrm{C}$ & 1 & 32.84 & 0.56 & 11.34 & 0.28 & 28.16 & $5.33^{*}$ \\
\hline $\mathrm{S} \times \mathrm{T}$ & 1 & 71.15 & 1.21 & 0.17 & 0.00 & 0.01 & 0.00 \\
\hline $\mathrm{C} \times \mathrm{T}$ & 1 & 233.49 & 3.81 & 1.26 & 0.03 & 0.13 & 0.02 \\
\hline $\mathrm{S} \times \mathrm{C} \times \mathrm{T}$ & 1 & 4.74 & 0.08 & 11.55 & 0.28 & 15.87 & 3.01 \\
\hline Error & 32 & 58.59 & & 40.85 & & 5.28 & \\
\hline Total & 39 & & & & & & \\
\hline
\end{tabular}

a: The numbers $(1,2$ and 8$)$ are those of adult pairs.

b: Mean of square.

${ }^{*}$ and ${ }^{* *}$ show significant differences at the $5 \%$ and $1 \%$ levels, respectively.

The analyses showed effects for each of three sources of variation (though not statistically significant in some cases). In almost all cases, no interactions among them were found. However, the interaction between strain and chromosome type $(\mathrm{S} \times \mathrm{C})$ was significant at density-8. This is due to the following: While the mean number of eggs laid by homozygous females was slightly larger than that laid by heterozygous ones in strain $10 \mathrm{~A}$ (this is exceptional), the situation was reversed in strain $22 \mathrm{~B}$. As shown in Table 2, the $\mathrm{C} \times \mathrm{T}$ interaction was also observed in density-1, though it was not significant. This may be due to the fact that the mean number of eggs laid by heterozygous females of strain $22 \mathrm{~B}$ was much larger under the constant temperature than under the fluctuating one.

Change of mean number of eggs laid for 20 days: The mean number of eggs laid for 20 days was analyzed by dividing the time into two 10 day periods. The mean 
Table 3. Mean number of eggs laid for the first 10 days $(a)$ and the last 10 days $(b)$ of oviposition, and the rate of change of mean number of eggs between two 10 day periods $(c)$

\begin{tabular}{|c|c|c|c|c|c|}
\hline \multirow{2}{*}{ Strain } & \multirow{2}{*}{$\begin{array}{l}\text { Temperature } \\
\text { condition }\end{array}$} & \multirow{2}{*}{$\begin{array}{c}\text { Chromosome } \\
\text { type }\end{array}$} & \multicolumn{2}{|c|}{ Mean number of eggs per female per day } & \multirow{2}{*}{$(c) \#$} \\
\hline & & & the first days $(a)$ & the last 10 days $(b)$ & \\
\hline $10 \mathrm{~A}$ & Constant & Homo & 39.80 & 28.83 & 27.56 \\
\hline$" \prime$ & " & Hetero & 43.61 & 35.17 & 19.35 \\
\hline$" \prime$ & Fluctuating & Homo & 35.17 & 29.54 & 18.31 \\
\hline$\prime \prime$ & " & Hetero & 38.84 & 33.47 & 13.83 \\
\hline $22 \mathrm{~B}$ & Constant & Homo & 32.24 & 19.90 & 38.28 \\
\hline " & " & Hetero & 40.05 & 25.62 & 36.03 \\
\hline$" \prime$ & Fluctuating & Homo & 27.13 & 18.81 & 30.67 \\
\hline " & " & Hetero & 31.78 & 22.41 & 29.48 \\
\hline
\end{tabular}

$\#: \quad(c)=\frac{(a)-(b)}{(a)} \times 100$.

Table 4. Proportions of mean number of eggs under the fluctuating temperature to that under the constant temperature $(F / C)$

\begin{tabular}{ccccc}
\hline Strain & Chromosome type & The first 10 day $(a)$ & The last 10 days $(b)$ & $(c) \#$ \\
\hline $10 \mathrm{~A}$ & Homo & $0.909 \$$ & 1.025 & 0.664 \\
$\prime \prime$ & Hetero & 0.891 & 0.952 & 0.715 \\
$22 \mathrm{~B}$ & Homo & 0.842 & 0.945 & 0.801 \\
$" \prime$ & Hetero & 0.794 & 0.875 & 0.818 \\
\hline
\end{tabular}

$\#: \quad(c)=\frac{(a)-(b)}{(a)} \times 100$.

\$: calculated from the data of Table 3 .

numbers of eggs for the first 10 days $(a)$, for the last 10 days $(b)$, and the percentage difference between these two, $\left[(c)=\frac{(a)-(b)}{(a)} \times 100\right]$ are shown in Table 3 .

The values of $(c)$ were calculated in order to ascertain the rate of change of mean egg number with time. Though it is not shown in Table 3, the values of $(c)$ increased with adult density, i.e., the rate of change of mean egg number between two 10 day periods was larger for the crowded conditions than for the uncrowded ones. As shown in Table 3, the mean number of eggs laid for the first 10 days was larger than that for the last 10 in all cases. This tendency was found to be more pronounced with the constant temperature than with the fluctuating one, and it is consistent with the results of the oviposition pattern mentioned above. In strain $10 \mathrm{~A}$, the $(c)$-value of homozygote was larger than that of heterozygote under any temperature condition. The relationship was the same in strain $22 \mathrm{~B}$.

The data from the three density levels were pooled, so that the mean egg number under the fluctuating temperature $(F)$ could be compared to that under the constant one $(C)$; to compare these two, the ratio- $F / C$ was calculated for both chromosome 
Table 5. Regression coefficients of mean number of eggs laid by homozygous and heterozygous females of strains $10 \mathrm{~A}$ and $22 \mathrm{~B}$ on time (in days) for 19 days (from the third to the 21st day after eclosion) under various conditions

\begin{tabular}{|c|c|c|c|c|c|}
\hline \multirow{2}{*}{ Temperature } & \multirow{2}{*}{ Density } & \multicolumn{2}{|c|}{ Strain $10 \mathrm{~A}$} & \multicolumn{2}{|c|}{ Strain $22 \mathrm{~B}$} \\
\hline & & Homo & Hetero & Homo & Hetero \\
\hline Constant & 우 $1 \times \hat{o} 1$ & -1.10 & -1.00 & -1.75 & -1.56 \\
\hline " & $2 \times \quad 2$ & -2.12 & -1.67 & -1.04 & -2.07 \\
\hline “ & $8 \times 8$ & -1.52 & -1.73 & -1.71 & -1.63 \\
\hline Constant & Pooled & -1.57 & -1.45 & -1.50 & -1.75 \\
\hline Fluctuating & 우 $1 \times \hat{o} 1$ & -0.88 & -0.36 & -0.60 & -1.37 \\
\hline " & $2 \times \quad 2$ & -0.99 & -0.87 & -1.35 & -1.17 \\
\hline$"$ & $8 \times 8$ & -1.31 & -1.15 & -1.33 & -1.22 \\
\hline Fluctuating & Pooled & -1.06 & -0.79 & -1.09 & -1.25 \\
\hline
\end{tabular}

types of each strain. The results are given in Table 4 .

In almost all cases, the ratio $(F / C)$ was less than one, i.e., the mean number of eggs was smaller under the fluctuating temperature than the constant one. Moreover, the decrease in egg production under the fluctuating temperature was more pronounced in heterozygous fles than in homozygous fles in any strain or period.

To demonstrate more clearly the rate of change of oviposition with time, the mean number of eggs laid by homozygous or heterozygous females was regressed on time, using 19 days as the time points (from the third to the 21st day after eclosion) under the various conditions. The regression coefficients are shown in Table 5, where they are compared with the $(c)$-values.

The absolute values of the regression coefficients under the constant temperature were always larger than those under the fluctuating one. This relationship was found for both homozygous and heterozygous flies of either strain. Also, the absolute values for homozygous flies were consistently higher than those for heterozygous ones at any density level in strain 10A. A similar situation was obtained in strain 22B. However, it was slightly reversed when the data were pooled over the three density levels; this is due to the reversal observed with density-2 under the constant temperature and density-1 under the fluctuating one (see Table 5). The regression coefficient from the crowded conditions seemed to be larger than those from the uncrowded ones.

These relationships among the regression coefficients do not serious, conflict with the oviposition patterns (Figs. 1 and 2) or the (c)-values shown in Table 3. From these analyses, it is clear that flies maintained in crowded conditions or under a constant temperature lay more eggs in the early part of the adult period, compared with those in uncrowded conditions or a fluctuating temperature. This tendency is more notable in homozygous flies than in heterozyous ones.

Mortality at the 38th day under various conditions: For the 20 days in which fecundity was tested, most of the flies remained alive, but they died gradually afterwards. In order to clearfy the relationship between fecundity and mortality, the latter (per- 
Table 6. Mortality (percentage of dead flies) at the 38th day after eclosion and the $(c)$-value under various conditions

\begin{tabular}{cccccc}
\hline \hline \multirow{2}{*}{ Temperature } & $\begin{array}{c}\text { Chromosome } \\
\text { type }\end{array}$ & \multicolumn{2}{c}{ Strain 10A } & \multicolumn{2}{c}{ Strain 22B } \\
\cline { 3 - 6 } & & Mortality & $(c) \#$ & Mortality & $(c) \#$ \\
\hline Constant & Homo & 51.8 & 27.56 & 70.0 & 38.28 \\
" & Hetero & 50.0 & 19.35 & 67.3 & 36.03 \\
Fluctuating & Homo & 35.5 & 18.31 & 75.5 & 30.67 \\
" & Hetero & 32.7 & 13.83 & 66.4 & 29.48 \\
\hline
\end{tabular}

$\#: \quad(c)=\frac{(a)-(b)}{(a)}-\times 100$, where $(a)$ and $(b)$ are the mean number of eggs for the first 10 days and for the last 10 days, respectively.

Table 7. Differences of mortality (percentage of dead flies) at the 38th day between sexes under various conditions

\begin{tabular}{lcccc}
\hline \multirow{2}{*}{ Temperature } & \multicolumn{2}{c}{ Strain $10 \mathrm{~A}$} & \multicolumn{2}{c}{ Strain 22B } \\
\cline { 2 - 5 } & Female & Male & Female & Male \\
\hline Constant & 46.36 & 60.91 & 67.27 & 69.09 \\
Fluctuating & 42.73 & 26.36 & 74.55 & 67.27 \\
\hline
\end{tabular}

centage of dead flies) was examined at the 38th day. The results pooled through three density levels are given in Table 6 along with the $(c)$-values.

The mortality of strain $22 \mathrm{~B}$ was higher than that of stran $10 \mathrm{~A}$ in all cases. In all strains and temperature conditions, the mortality of homozygous flies was higher than that of heterozygous ones. Moreover, flies did earlier under the constant temperature than under the fluctuating one. These results suggest a relationship between mortality and the $(c)$-values obtained when the three density levels are pooled, as they are in Table 6. Therefore, the coefficient of correlation was calculated. The coefficient $(r=$ 0.90$, d.f. $=6)$ was significant at the $1 \%$ level. In other words, it means that the flies which laid more eggs in the early part of the adult period died earlier. Thus, the oviposition curve was related to mortality. However, no correlations between mortality at the 38th day and the mean number of eggs laid for 20 days were found $(r=-0.08$, $d . f .=22$, as based on the data of the mean values of flve replicates).

It is interesting to examine whether there is a difference in the mortality of the two sexes. It is said that in general the mortality of males is higher than of females in Drosophila. In this experiment, such a relationship was observed only under the constant temperature (Table 7). The relationship was reversed under the fluctuating temperature, i.e., females had higher mortality than males.

\section{DISCUSSION}

In insects such as Drosophila, fecundity (egg production) is an important component 
of adult fitness. As mentioned earlier, it is strongly influenced by various environmental factors.

Fecundity is known to be affected by adult density and by nutritional conditions (Furuya and Mori 1961); however, Karandinos and Axtell (1972) reported no density effects on the birth rate (the number of newly hatched offspring) of Hippelelates pusio. In the present experiment, the fecundity of $D$. melanogaster was greatly influenced by adult density. In other words, the mean number of eggs per female laid by one or two females was more than that laid by eight. However, there was no significant difference between the mean number of eggs laid by one female and that laid by two, though at face value the difference was negative. Therefore, it could not be determined for sure whether fecundity was Allee-type ${ }^{1)}$ or Drosophila-type ${ }^{2>}$ in the language of Karandinos and Axtell (1972).

Egg production per female per day was suppressed in the highest density. This may be explained in the following way: In a limited space, the number of eggs laid by flies does not exceed an upper limit. Therefore, even if many flies are introduced into a limited space, the number of eggs does not increase along with the number of flies. However, the mechanism whereby fecundity diminishes is unknown. The problem should be examined in more detail.

In this experiment, it was found that the fecundity of flies was noticeably affected by the temperature condition, i.e., the mean number of eggs was smaller under the fluctuating temperature than under the constant one. This agrees with the result of Oshima and Kawanishi (1969). I believe that it is caused by the inhibition of oviposition at the lower and higher temperatures under the fluctuating condition.

In any 10 day period, the decrease of egg production under the fluctuating temperature was larger for heterozygous flies than for homozygous ones (Table 4). This result cannot be explained by the stability of heterozygotes in the face of environmental variability. Also, it was found that mean number of eggs laid by heterozygous flies was larger than that laid by homozygous ones (Table 1). The difference may be mainly caused by the high fecundity of the $C y$-chromosome, i.e., the difference of genetic system affecting fecundity between chromosome types. Furthermore, a difference in fecundity between strains was observed in this experiment. This suggests that there is variation for genes controlling fecundity in natural population.

In order to indicate the rate of change of egg production with time, the $(c)$-values were calculated for the various conditions. In either strain, the $(c)$-values were larger for the crowded condition or under the constant temperature than for the uncrowded conditions or under the fluctuating temperature. Moreover, the values were larger for homozygous flies than for heterozygous ones. In particular, attention should be directed to the daily egg production (oviposition pattern) examined under the fluctuating temperature. Daily egg production and the $(c)$-value were smaller under the fluctuating temperature than under the constant one. In other words, the flies laid less eggs, but

1) Allee-type: The rate of oviposition is low at low population density, high at middle population density and low at high population density.

2) Drosophila-type: The rate of oviposition is highest at low population density and decreases as its density increases. 
continued to lay eggs over a larger period under the fluctuating temperature. Such flies may be more adapted to a variable natural environment, since egg production is spread over time and space. It is notable that the results obtained in this experiment are similar to those of Oshima and his associates $(1971,1972,1974)$ with respect to periodical light and dark conditions.

It appears that mortality can be predicted from the oviposition curve (regression coefficient): Flies which show large negative values of the oviposition regression coefficient die early. In order to document this, mortality at the 38th day after eclosion was examined in this experiment. The results showed that mortality was positively correlated to the $(c)$-value. However, mortality was not related to mean number of eggs for 20 days. This suggests that flies which laid the most eggs in 20 days did not always die early. For a more detailed analysis, egg production over the entire life span should be examined.

In this experiment, it was found that mortality was higher under the constant condition than under the fluctuating one. This result is similar to that of Erk and Samis (1970), who found that life span is longer under alternating light and dark conditions than those of constant light.

The mortality was different between the sexes. The difference was closely related to a temperature condition. Namely, under the constant temperature, the mortality of males was higher than that of females. On the other hand, under the fluctuating temperature, the mortality of males was lower than that of females. If such a relation is universal in many organisms, it is biologically significant and interesting.

\section{SUMMARY}

By using homozygous or heterozygous flies of two strains of Drosophila melanogaster, the effect on fecundity (egg production) of adult density and fluctuating temperature were investigated. The results indicated that: (1) Fecundity was strongly influenced by adult density and by the temperature condition, i.e., egg production was lower in the higher density and under the fluctuating temperature $\left(a 20^{\circ} \mathrm{C} \sim 30^{\circ} \mathrm{C}\right.$ cycle per day) in both strains. (2) The mean number of eggs laid by heterozygous flies was larger than that laid by homozygous flies. However, the decrease in oviposition under the fluctuating temperature was more pronounced for heterozygous flies than for homozygous ones of both strains. (3) In order to examine the change of the daily egg production, the regression coefficients of mean number of eggs on day and the $(c)$-values were estimated under various conditions. The $(c)$-value and the absolute value of the regression coefficient were larger for homozygous flies, in the crowded condition and under the constant temperature than for heterozygous ones, in the uncrowded conditions and under the fluctuating one, respectively. (4) Mortality was related to the coefficient of regression of daily egg production on time. Therefore, mortality (longevity) can be predicted from this coefficient. Also, mortality was different between the sexes, and the difference was affected by temperature. (5) The effect of the fluctuating temperature on fecundity and mortality was similar to that of fluctuating light on them. Some problems arising from the results were discussed. 


\section{ACKNOWLEDGMENT}

The author is greateful to Dr. Chozo Oshima of the National Institute of Genetics for his encouragement and his critical reading of the manuscript. The author is also indebted to Prof. Koichiro Tsunewaki, Laboratory of Genetics, Kyoto University, for his kind guidance for his carefully checking of the manuscript. The author would also like to acknowledge Drs. Michael Simmons of the Department of Genetics and Cell Biology, University of Minnesota, and Ohmi Ohnishi, Laboratory of Genetics, Kyoto University, for their kindness in reading the manuscript. The author also is thankful for the helpful criticism given by Dr. Takao Watanabe and his colleagues of the Department of Physiological Genetics, the National Institute of Genetics.

\section{LITERATURE CITED}

Beardmore, J. A., and L. Levine, 1963 Fitness and enviromental variation. I. A study of some polymorphic populations of Drosophila pseudoobscura. Evolution 17: 121-129.

Choo, J. K., and C. Oshima, 1974 Phototactic selection and its effects on some quantitative characters of Drosophila virilis. Environ. Control in Biol. 12: 41-51.

Dawood, M. M., and M. W. Strickberger, 1964 The effect of larval interaction on viability in Drosophila melanogaster. I. Changes in heterozygosity. Genetics 50: 999-1007.

Erk, F. C., and H. V. Samis, Jr., 1970 Light regimens and longevity. Drosophila Inf. Serv. 45: 148

Furuya, Y., and S. Mori, 1961 Influence of larval environments on the life of Drosophila melanogaster. Jap. J. Ecol. 11: 104-111.

Karadinos, M. G., and R. C. Axtell, 1972 Population density effects on fecundity of Hippelelates pusio Loew. (Diptera: Chlopidae). Oecologia 9: 341-348.

Lewontin, R. C., 1955 The effects of population density and composition on viability in Drosophila melanogaster. Envolution 9: 27-41.

Lewontin, R. C., and Y. Matsuo, 1963 Interaction of genotypes determining viability in Drosophila busckii. Proc. N. A. S. 49: 270-278.

Ohba, S., 1961 Analytical studies on the experimental population of Drosophilla. I. The effect of larval population upon the pre-adult growth in D. melanogaster and D. virilis, with special reference to their nutritional conditions. Biol. J. Okayama Univ. 7: 87-125

Ohnishi, S., 1976 Effects of population density and temperature condition on fitness in Drosophila melanogaster. I. Developmental time and pre-adult viability. Japan. J. Genetics 51: 19-25.

Oshima, C., and M. Kawanishi, 1969 Adaptation of Drosophila melanogaster under the constant and fluctuating temperatures. Environ. Control in Biol. 7: 21-29 (in Japanese with English summary).

Oshima, C., and J. K. Choo, 1971 Circadian rhythm of fecundity of Drosophila virilis under various light environments. Ann. Rep. Nat. Inst. Genet. 22: 105-107.

Ushima, C., K. Inoue, and S. Ishiwa, 1972 Influence of light and dark environments on the oviposition of Drosophila virilis. Environ. Control in Biol. 10: 187-191 (in Japanese with English summary).

Palabost, L., 1972 The influence of density on the larval viability of Drosophila melanogaster. Drosophila Inf. Serv. 49: 118.

Parsons, P. A., 1959 Genotypic-environmental interactions for various temperatures in Drosophila melanogaster. Genetics 44: 1325-1333.

Young, S. S. Y., 1970 Direct and associate effects of body weight and viability in Drosophila melanogaster. Genetics 66: 541-554. 